\title{
Christina R. Victor (2010). Aging, Health and Care. Bristol: The Policy Press, 224 pp. ISBN 9781847429 (paperback)
}

\author{
Reviened by Duane A. Matcha*
}

One of the major topics of investigation today is that of the ongoing population shift towards an older age. This shift reflects decades of improved public health and changing family patterns that have resulted in increased life expectancy as well as decreasing fertility rates throughout the world. This transition has brought about significant changes in generational relationships as well as questions regarding the viability of societies providing necessary services to a rapidly growing older-age population. In particular, there is a growing and misplaced concern that the rising cost of health services is primarily the result of an everexpanding older age population. Many of the misconceptions surrounding this population shift are promulgated by a media that has little knowledge of demography and gerontology. It would be wise for those in the media, particularly in Great Britain, who write about aging issues, to read this book.

Victor, a gerontologist, provides the reader with a broad overview as well as an in-depth analysis not only of the demographic characteristics of the older age population in Great Britain, but also of their needs. Although her focus is primarily on the aging population in Great Britain, she broadens the scope to include other industrialized countries, when appropriate. Victor's book adds to the growing list of efforts to frame our

* Duane A. Matcha, Department of Sociology, Siena College, Loudonville, NY, USA 
International Journal of Ageing and Later Life

understanding of who the elderly are and what we know about them. While the book covers familiar territory, it does so with crisp writing, extensive use of current data, and excellent learning tools that expand the knowledge base of the reader. The identification of websites specific to the chapter material increases the viability of the book as an international source of the study of aging.

Although relatively short at 224 pages, the book does not compromise on substance. The chapters address the primary areas generally associated with an analysis of an older age population. The introductory chapter on key concepts is a useful starting point as it frames the broad issue of population aging and its consequences for society. Additional chapters address health and mortality, chronic disease and disability, mental health and psychological well-being, consumption and health, provision and utilization of services, and health in the future.

The core of the book is built around four interrelated points that include:

- Providing an overview of the health status of older people.

- Understanding how older people define and understand the meaning of health in old age.

- The nature and extent of health problems in old age and the policy and service response to the health issue of older people.

- Looking at health at a population level and examining the variability of health experiences in later life with key elements of social structure, most notably gender, age, socioeconomic status and ethnicity (p. 3).

With regard to the first point of providing an overview of the health status of older people, the book does an excellent job. In Chapter 1, Victor outlines key concepts of health and then moves into Chapter 2 in which these concepts are applied to the aging population. Throughout the book, Victor returns to this point and uses it as a basis for examining health, mortality, chronic disease and disability.

The second focus of the book, understanding how older people define and understand the meaning of health in old age, offers a standard analysis of the relationship. Here, Victor discusses the medical and sociological models of health, identifies a number of factors that interact to define health in old age (p. 38), and addresses them in depth. 
Aging, Health and Care. Book Review

The third focus of the book, that of the nature and extent of health problems in old age is particularly important as it demystifies the relationship between age and health. Again, the use of data offers an objective analysis of the relationship.

The fourth point is perhaps the most important as it moves us away from the assumption that being old is the primary consideration when examining health issues. Rather, the variability between sociodemographic factors such as sex, race, and income levels provides a further disaggregation of facts and illustrates the impact that other factors play in determining the health of an older person. Here, the book excels in its wealth of data.

Perhaps the most interesting chapter is that of Consumption and Health. Here, Victor offers an interesting direction for the research of aging populations. Her discussion of the percentage of income that older persons in the UK spend on health care then contrasted to the United States opens opportunities for discussion of health policy implications. Also of interest is the amount of money spent on lifestyle consumption by older persons. This area generally focuses on anti-aging products but also includes expenses for, among other items, gym membership and clothing. Given the general lack of coverage or more importantly, positive coverage in the media, research of consumption patterns and health offers a new and dynamic path to understanding health differences and consequences among this population.

Finally, the learning tools that Victor incorporates throughout the book are welcome. For example, she provides boxed inserts of key points at the beginning and end of every chapter. She also incorporates boxed activities in which questions are posed regarding the topic being addressed. These questions are useful as they direct attention to specific information within the chapter. At the end of every chapter, Victor provides a number of web links specific to the topic along with further readings and activities. In total, the book offers an excellent in-depth examination of the health of the elderly in Great Britain with some international comparisons. 